\title{
STEPWISE ORIGIN AND FUNCTIONAL DIVERSIFICATION OF THE AFL SUBFAMILY B3 GENES DURING LAND PLANT EVOLUTION
}

\author{
YANG $\mathrm{LI}^{*}$, KE $\mathrm{JIN}^{\dagger}$, ZHU ZHU ${ }^{\ddagger}$ and $\mathrm{JI} \mathrm{YANG}^{\S}$ \\ Center for Evolutionary Biology \\ School of Life Science, Fudan University \\ Shanghai 200433, P. R. China \\ *082023106@fudan.edu.cn \\ †inke@fudan.edu.cn \\ $\ddagger_{z z h u @ f u d a n . e d u . c n}$ \\ §iziang@fudan.edu.cn
}

Received 15 July 2010

Revised 25 August 2010

Accepted 10 September 2010

\begin{abstract}
The AFL genes (ABI3/VP1, FUS3 and LEC2) belong to the plant-specific B3 superfamily, playing important roles in regulating seed development and maturation. It is unclear, however, whether these genes appeared at the same time as the origin of seed plants and if all these genes are necessary and sufficient for seed development for all seed plants. By conducting a genome-wide comparative analysis of the putative AFL genes in various plant species, we found that the $A B I 3$ homologous genes existed in all land plant genomes, but the FUS3 homologous were present only in seed plant genomes and the LEC2-like sequences only in dicot genomes. Phylogenetic analysis indicated that the AFL genes had undergone successive rounds of gene duplication and subsequent diversification during land plant evolution, resulting in the stepwise origin of the ABI3, FUS3 and LEC2 genes. Comparison of gene structure of the AFL genes revealed a trend of decreasing in the number of conserved domains from ABI3 to FUS3 and LEC2.
\end{abstract}

Keywords: AFL B3 genes; genome-wide comparative analysis; stepwise origin; functional diversification.

\section{Introduction}

B3 genes encode plant-specific transcription factors, which play a variety of roles in plant growth and development. More than one hundred B3 genes have been identified from various plant genomes. Proteins encoded by B3 genes vary in sequence similarity and domain structure but all contain a highly conserved B3 domain, forming the B3 superfamily. This superfamily consists of several distinct families, including the LAV, ARF, RAV and REM families, with a few subfamilies to be identified in each family. ${ }^{1,2}$ The AFL subfamily belongs to the LAV family, containing three members: ABSCISIC ACID INSENSITIVE 3 (ABI3), FUSCA 3 (FUS3) and 
LEAFY COTYLEDON 2 (LEC2). The three members of the AFL subfamily play important roles in regulating seed development and maturation. ${ }^{3-5}$ Loss-of-function mutants in ABI3, FUS3 and LEC2 exhibit defects in seed maturation and desiccation intolerance. ${ }^{6,7}$ ABI3, FUS3 and LEC2 are thus considered master genes in controlling seed development, especially for seed maturation. ${ }^{8-11}$

Seed formation is one of the key innovations during land plant evolution, ${ }^{12}$ which endows plants with higher adaptability toward unfavorable environment and strengthens the ability of dispersal and competition. The process of seed development can be divided into two phases, i.e. the early embryo morphogenesis phase and the late maturation phase. ${ }^{13,14}$ During the late maturation phase, the embryo accumulates various storage compounds and acquires desiccation tolerance, and eventually enters dormancy. ${ }^{15,16}$ The AFL B3 genes act in a partially redundant manner during seed maturation. ${ }^{9}$ It remains unclear, however, whether all these genes are necessary and sufficient for induction of the maturation phase of seed development for all seed plants. The questions such as whether these genes appeared at the same time as the origin of the seed plants and how they originated remain unanswered.

The $A B I 3$ gene was first identified from the maize genome, ${ }^{17}$ and is now found to be widely distributed in different plant genomes. There is currently limited information on the distribution patterns of FUS3 and LEC2, though their functions have been thoroughly studied in Arabidopsis., ${ }^{4,5}$ ABI3, FUS3 and LEC2 are closely related with each other in gene sequences and functions. However, the evolutionary relationships among ABI3, FUS3 and LEC2 are still ambiguous. We have ever explored the origin and evolution of another master regulatory gene for seed development, $L E C 1,{ }^{18}$ and revealed that, although $L E C 1$ is crucial to seed maturation, it is not seed-plant specific. The $L E C 1$ gene originated in non-seed vascular plant genomes and was inductively expressed under drought stress in nonseed plants. This gene was recruited to a novel regulatory network in the early stages of seed plant evolution and steadily expressed during seed development and maturation. ${ }^{9-11}$ It is thus intriguing to know whether this pattern of evolution is also applicable to the AFL B3 genes.

In this study, genome-wide screens for the AFL genes were performed based on currently available plant genome sequences. By conducting a comprehensive analysis of the distribution patterns, gene structures and phylogenetic relationships of ABI3, FUS3 and LEC2, we intend to address questions, such as when these genes originated, how they duplicated and diverged during evolution, and whether all these genes are indispensable components of the regulatory network involved in the control of seed development for all seed plants. We found evidence that the AFL genes experienced independent gene duplication events at different stages of land plant evolution. The FUS3 and LEC2 genes appeared in the gymnosperm and angiosperm genomes in a stepwise pattern via neo- and/or sub-functionalization of duplicated genes. 


\section{Methods}

\subsection{Genome-wide screen for putative AFL genes}

Two BLAST ways, BLAST via a web interface and stand-alone BLAST, were used to search for putative AFL genes in various land plant genomes. Since the structure and function of Arabidopsis thaliana ABI3, FUS3 and LEC2 genes have been most thoroughly characterized, we first downloaded the CDS and protein sequences of Arabidopsis AFL genes from TAIR (http://www.arabidopsis.org/), and then conducted extensive similarity searches through the public BLAST interfaces at the NCBI website and elsewhere, using Arabidopsis AFL sequences as query sequences. Multiple BLASTN and TBLASTN searches were carried out against the complete or nearly complete plant genome sequences in GenBank (http://www.ncbi.nlm.nih.gov/genomes/PLANTS/PlantList.html) and other public trace archives (http://www.plantgdb.org/, http://www.phytozome.net/), with a cutoff e-value of $\mathrm{e}^{-10}$. Since it has been revealed that sequences containing more than one structural/functional domain are problematic when used in homology searches, e.g. they can cause an explosion of a search to common domains, all sequences retrieved from GenBank and other databases were subjected to domain structure analyses using the SMART (http://smart.emblheidelberg.de/) and Inter-ProScan (http://www.ebi.ac.uk/InterProScan/) platforms, and compared with the structure of Arabidopsis AFL genes. Sequences with clearly distinct domain compositions from Arabidopsis AFL genes were excluded from the dataset, though possessing the highly conserved B3 domain.

To search for putative AFL genes in plants that are important for detecting the phylogenetic patterns of the AFL genes but their complete genomes are currently unavailable, e.g. Pinus taeda and Picea abies, BLAST searches were also performed against the available EST databases at http://plantta.jcvi.org/ and http://compbio.dfci.harvard.edu/tgi/. The conserved domain sequences of Arabidopsis AFL genes were used as query seeds to conduct BLASTN and TBLASTN searches. The acquired EST clusters were assembled using CAP3 ${ }^{22}$ to form consensus sequences, and then translated into putative peptide sequences using a 6 -frame translation scheme. By matching similarity of translation products to Arabidopsis target proteins, we identified potential homologous AFL sequences from plants without genome sequence information.

For conducting more thorough homology searches and refining the BLAST hits obtained from homology searches via web interfaces, stand-alone BLAST was also performed against the local databases (see Supplementary Material). The Formatdb program implemented in stand-alone BLAST 2.2.13 software ${ }^{23}$ was used to construct a BLAST database for each plant. BLAST searches were then performed on the stand-alone BLAST server, using Arabidopsis AFL sequences as queries. 


\subsection{Sequence alignment and phylogenetic analysis}

Multiple sequence alignments were generated with Clustal X 2.0.12 (24, 25) with default parameters: Gonnet series as the protein weight matrix and IUB as the DNA weight matrix, gap opening 10.00, gap extension 0.20 , delay divergence sequences $30 \%$, DNA transition weight 0.50, and adjusted manually with BioEdit. ${ }^{26}$ The AFL subfamily comprises diverse multi-domain genes. While the functional/structural domains are well conserved, the N/C-terminal extensions and/or internal insertions are highly variable among members of the family. We thus initially performed a global alignment of all sequences of interest, and conducted a preliminary phylogenetic analysis by constructing a neighbor-joining (NJ) tree using MEGA 4.1 ${ }^{27}$ to determine the number and composition of different groups. The sequences of each subgroup were then aligned again separately, and the sequences that could not be aligned unambiguously were removed. The final alignment was generated using Gblocks 0.91b, ${ }^{28}$ allowing smaller final blocks.

Phylogenetic analyses were conducted using the neighbor-joining (NJ), maximum parsimony (MP) and maximum likelihood (ML) methods. NJ and MP trees based on amino acid sequences were built using MEGA 4.1 and PAUP4.0b8, ${ }^{29}$ respectively. The Jones-Taylor-Thornton (JTT) model ${ }^{30}$ was employed for distance calculations in NJ analyses with pairwise deletion option selected. Heuristic tree search under parsimony was conducted using the tree-bisection-reconnection (TBR) swapping algorithm. The ML tree was constructed using RAxML, ${ }^{31}$ which is a program for sequential and parallel maximum likelihood-based inference of phylogeny. GTRCAT_GAMMA and PROTCAT_GAMMAIWAG were used as the models of nucleotide substitution and amino acid substitution, respectively. The robustness of the tree topology was assessed by bootstrap analysis, with 100 replicates for the ML method.

\section{Results}

\subsection{Phylogenetic distribution of the AFL genes in different lineages}

BLAST results showed that the AFL subfamily genes are widely distributed in various plants (Table 1). Three sequences homologous to the AFL B3 genes were identified from Physcomitrella patens and Selaginella moellendorffi genomes, respectively. Gene structure and phylogenetic analyses indicated that all these sequences are homologues of $A B I 3$. Of them, three $P$. patens genes $(P p A B I 3 A$, $P p A B I 3 B$ and $P p A B I 3 C$ ) have been functionally clarified. ${ }^{32}$ There is currently no complete genome sequence for gymnosperms. However, there exist a few databases that contain plenty of ESTs and incomplete genome sequences from various gymnosperms. We thus searched for the gymnosperm AFL genes against all the databases available. An ABI3 homologous gene was identified from Pinus taeda and Picea abies, respectively, which showed high similarity in gene sequence and 
Table 1. Identification and distribution patterns of AFL subfamily members of various plants. Numbers in the table stand for the copy number of each gene in various plant genomes. The last column summarizes total numbers of AFL subfamily members. As for Picea abies and Pinus taeda, because of the lack of EST data, some of the genes are not applicable (N/A).

\begin{tabular}{llccccc}
\hline Species name & Classification & ABI3 & FUS3 & LEC2 & IDEF1 & Total \\
\hline Physcomitrella patens & Bryophytes & 5 & 0 & 0 & 0 & 5 \\
Selaginella moellendorffii & Lycophytes & 4 & 0 & 0 & 0 & 4 \\
Picea abies & Gymnosperm & 1 & N/A & 0 & 0 & N/A \\
Pinus taeda & Gymnosperm & 1 & 1 & 0 & 0 & N/A \\
Arabidopsis thaliana & Dicot & 1 & 1 & 1 & 0 & 3 \\
Poplus trichocarpa & Dicot & 1 & 2 & 2 & 0 & 5 \\
Glycine max & Dicot & 2 & 2 & 1 & 0 & 5 \\
Manihot esculenta & Dicot & 2 & 2 & 1 & 0 & 5 \\
Oryza sativa & Monocot & 1 & 1 & 0 & 3 & 5 \\
Sorghum bicolor & Monocot & 1 & 1 & 0 & 2 & 4 \\
Zea mays & Monocot & 1 & 1 & 0 & 3 & 5 \\
\hline
\end{tabular}

structure with other annotated $A B I 3$ genes. We also found an incomplete homologue of FUS3 in P. taeda. The homologous ABI3 and FUS3 genes were present in all angiosperm genomes. However, the LEC2-like sequences were only found in the dicot genomes. BLAST searches of the cereal genomes also detected another group of conserved genes in Oryza sativa, Zea mays and Sorghum bicolor, that share sequence similarity with the angiosperm FUS3 and LEC2 but formed an independent clade in the phylogenetic tree. According to the genome annotation of $O$. sativa, these genes were homologues of IDEF1, encoding the iron deficiencyresponsive cis-acting element binding factors. ${ }^{33,34}$

\subsection{Phylogenetic relationship of $A F L$ genes}

The evolutionary relationships among various AFL genes were inferred by NJ, MP and ML analyses. Complete gene sequences and the B3 domain sequences were used to construct phylogenetic trees, respectively. The tree topologies produced by different methods were similar on the overall structure but not perfectly match. Figure 1 shows the phylogenetic tree reconstructed by ML using the B3 domain sequences. It highlights three important features in all inferred trees. First, the ABI3 genes of seed plants formed a well-supported group in the phylogenetic tree and showed a close relationship with those from non-seed plant genomes. Second, the FUS3 and LEC2 genes, as well as the IDEF homologous genes of angiosperms grouped into three separate clades, with the position of the IDEF group varying relative to other two groups in different trees. Third, the homologous FUS3 gene of P. taeda did not cluster together with the angiosperm FUS3 genes but formed an independent branch ancestral to the angiosperms genes except ABI3. 


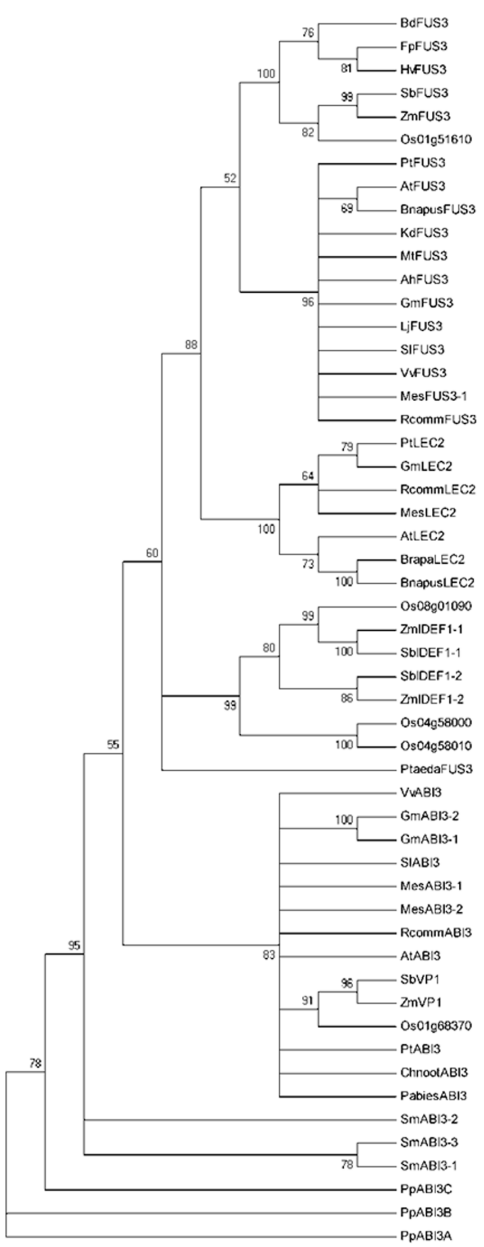

(a)

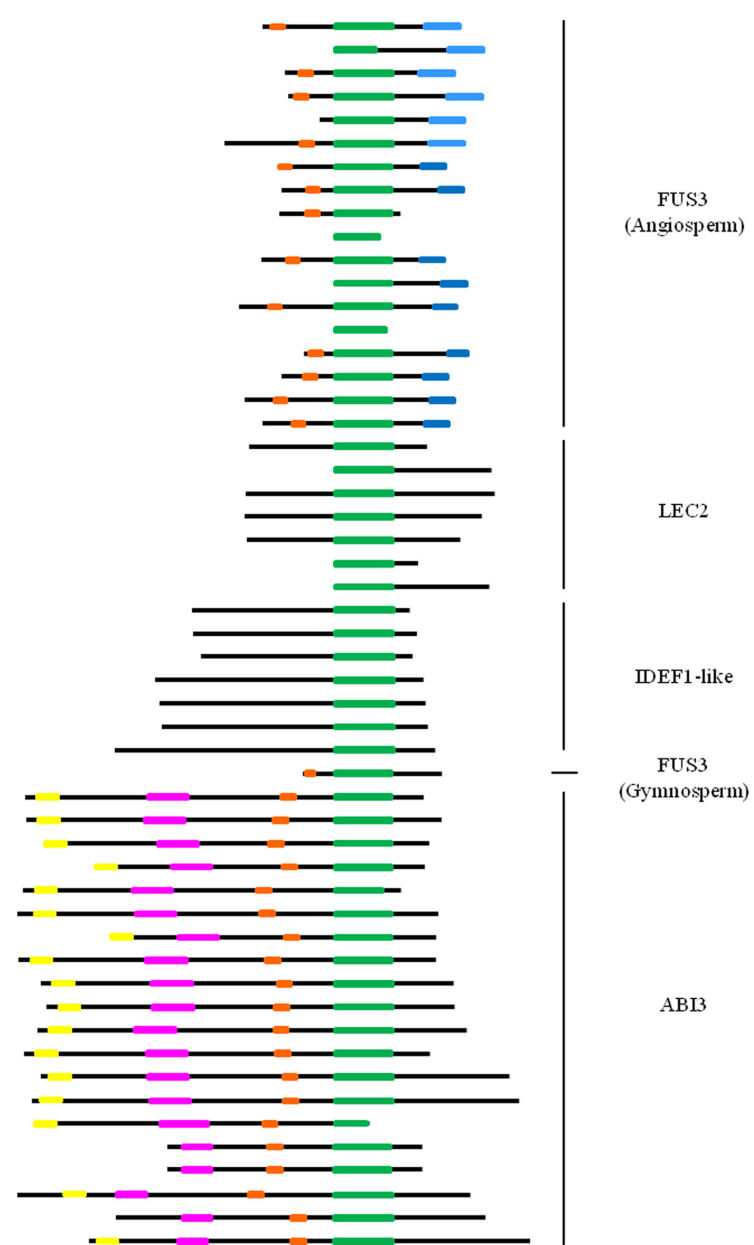

(b)

Fig. 1. (a) Phylogenetic analysis of protein sequences of B3 domain from AFL family of 23 species representing all plant groups from moss to seed plants. The species is indicated by the first two letters of the gene names. Ah: Arachis hypogaea; At: Arabidopsis thaliana; Bd: rachypodium distachyon; Brapa: Brassica rapa; Bnapus: Brassica napus; Chnoot: Callitropsis nootkatensis; Fp: Festuca pratensis; Gm: Glycine max; Hv: Hordeum vulgare; Selmo: Selaginella moellendorffii; Kd: K.daigremontianas; Lj: Lotus japonica; Mes: Manihot esculenta; Mt: Medicago truncatula; Os: Oryza sativa; Pabies: Picea abies; Pp: Physcomitrella patens; Pt: Populus trichocarpa; Ptaeda: Pinus taeda; Rc: Ricinus communis; Sb: Sorghum bicolor; Sl: Solanum lycopersicum; Vv: Vitis vinifera; Zm: Zea mays. (b) Domain position and composition of each AFL genes. Different domains are indicated with different colors. Yellow block: A (serine-rich) domain; Purple block: B1 domain; Orange block: B2 domain; Light blue block: FUS3-specific conserved sequence of monocots; Dark blue block: FUS3-specific conserved sequence of dicots. 

MesFUS3-1 -

PtFUS3 (

MesFUS3-2 --

GmFUS3 - 3 (-

MtFUS3 -

VVFUS3 -

SIFUS3 -

AtFUS3 ASTLVASVD---1.-- 39 RCFUS3 -CTWITSGDSVTE---EGDNKANN------TFEEASSDQ-------GSGRTVMMTRSSGDDD------NVVAVL 65 MesFUS3-1 QATLKSKHTQQSEKAKKGKGKERKRRLGRLMMKEQA.AVHEKPEARAWVAGGDGVAAEGDKVRGGSDRSWVGHGGDLVSMV 95

PtFUS3 -

MesFUS3-2 --

GmFUS3 TLCVCVCVSKKKNPKLMMMDPRQREK--LLHKTEACAFVAGVVPELSLVTVPGNNNNTMNVNNNNNN-16

MtFUS3 - 3 -

VVFUS3 - 3 -

SIFUS3 - 3 -

AtFUS3 GVNWK-KRRMPRQRRSSS---SFN-LLSFP----------PPMPPISHVP-------TPLPARKIDPRKLRFLFQKEL 95

RCFUS3 GFSVIRKRRMPRQRRPSS---SINNLFSFPIATNASSSSSSTTTTTTSHVP------DLPSFPARVIDQRRLSFLFQKEL 136

MesFUS3-1 GFDVKRKKRMPRQRRQSS---SINYLFSFPNTVTVSSSCCSSSAATTSHVPSSSPLHHLPSFPTRVIDQRRLSFLFQKEL 172

PtFUS3 GFEIKR KRMPRQRRSSS---TINHLLSF--AANASCSATTHLHVPAFSLP----LQDPSSLPA RVIDPRRLRFLFQKEL 73

MesFUS3-2 ----

GMFUS3 HSQSHRRKRMARQRRSTNPTLLMNPLINNN-NNKSGSSLPSPSTASSSHVP----LSSSTLPPAREIDQRRLRFLFQKEL 191

MTFUS3 FGTVHR KRMARQRRSSSTITIH-LKNLP-SSTTTTTTTTSHVPISPIP----PLFHSLPPA REIDHRRLRFLFQKEL 131

VVFUS3 SFGVQGEKRMPRQRRSSA---IN-LLTFA---1

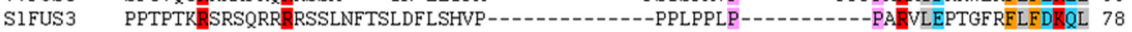

AtFUS3 KNSDVSSLRRMILPKKAAEAHLPALECKEGI-PIRMEDLDGFHVWTFKY-RYWPNNNSRMYVLENTGDFVNAFGLQLGDF 173

RcFUS3 KNSDVSSLKRMVPKRAAEAHLPVLESKEGI-FISMDDLDGLHVWSFEY-RYWPNNNSRMYVLENTGDFVNIHGLQLGDF 214

MesFUS3-1 KNSDVSSL KRMILPKKAAEAHLPILESKEEI -FISMDDLDGLHVWSFKY -RYWPNNNSRMYVLENTGDFVNIḦGLQLGDF 250

PtFUS3 QNSDVSSLRRMLPKKAAEVHLPFLESEEGI -FISMDDLDGL HVWSFEYSRYWPNNNSRMYVLENTGDFVNA HELLGDF 152

MesFUS3-2 KNSDVSSLKRMILPKKAAEAHLPLLESKEGI -FISMDDLDGMHVWSFKY-RYWPNNNSRMYVLENTGDFANI ḦGLQPGDF 138

GmFUS3 KNSDVSSLRRMILPKKAAEAFLPALESKEGI -VISMDDIDGLHVWSFKY-RFWPNNNSRMYVLENTGDFVNTHGLRFGDS 269

MtFUS3 KNSDVSSLRRMLPKKAAEAFLPVLESEEGI-LLSMDDLDGLHVWSFKY-RFWPNNNSRMYVLENTGDFVSTHGLRFGDS 209

VVFUS3 KNSDVGSLRRMVPKKSAETHLPLLEA KEGI-LITMYDLDGQHVWNFEY-RFWPNNNSRMYVLENTGEFVNVHGLQLGDY 177

S1FUS3 QNSDVSSLRRIVVPKKAAERYLPALEI KEGE-PITMDDMDGIHVWSFRY-RYWPNNSSRMYVLENTGDFVQTHELRQGDY 156

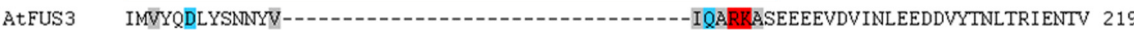

RcFUS3 IMVYRDDQNQNYV----- 3 -

MesFUS3-1 IMVYKDDDQNQNYV--

PtFUS3 IMVYQDSQSQNYV------

MesFUS3-2 IMVYKDNLNQNYVSRAPFQNLLHVKIPSFNWFPSRFKLEISDT--QVIQAKKASDED----------VYANITLTE--- 202

GmFUS3 ILVYQDSENNNYV---

MtFUS3 IMVYQDNQNHNYV---- 3 -

VvFUS3 IMLYHDGQTQSLV-_-_-_-_-_- 3

SIFUS3 FALHYNDQKQIYG--_-

AtFUS3 VNDLLLQDFNHHMNNNNNNSNSNSNKCSYYYPVIDDVTTNTESFVYDTTALTSNDTPLDFLGGHTTTTN----NYYSKFG 295 RCFUS3 VNDIVLYDC--------EINKSSSFYMNYPIVDNTG---LSFIYDTIT-FSDDSPLDFLGGSLTN-------YSRIG 304 MesFUS3-1 VSDMSLNDYD-------QVNKCSSFYMNYPMVDNTG---LSF IYDTTTNFSDDSPLDFWGGSLTN------YSRMG 342 PtFUS3 VNDTVLHDY---------EVNKFSSFYVNYPVVDNTG---LSFIYDTTT-FSNYSPLDFLGGSMTN---.----FSRIG 242 MesFUS3-2 VDDIFLHDFK--------EINKSSFFNMNYPIVDNTG---LSFIYDNIT-FSDDSPLDFLGGSLTN-------YSRIG 261 GmFUS3 INDIFLNDY--------EVNKPGCFNVTYPAVNDTG---MSFIYETIF--SNNDSPLDFLGGSMTN-------FSRIG 358 MtFUS3 INHIFVDDY---------EVNKS-CFDVAYPAMNDTS---MSF IYDTTI --SNDDSPLDFLGGSMTN-------YSRIG 297 VVFUS3 VSDLFLQDL--------EANRS-----NYFLAMDTG---TSFVYETIF--SNDSPLDFLGGSMTNYPGGSVSNYSRFG 270 S1FUS3 TDYAQAADDG----NGALMNEAEMDMSSFYFPAMDNEMG--MSF IYDTSF--WNEPAFDFVWGPMTY------YSTNV 255

$\begin{array}{lll} & & \\ \text { AtFUS3 } & \text { TFDG-LGSVEN-ISLDDFY------- } & 312 \\ \text { ReFUS3 } & \text { HRET-FGPVES-LSLDEFN-------- } 321 \\ \text { MesFUS3-1 } & \text { HMENSFGSVES-FSLDEFN-------- } 360 \\ \text { PtFUS3 } & \text { HLES-FGSVEN-MSLDDFY-------- } 259 \\ \text { MesFUS3-2 } & \text { HMES-FGSVES-LSLDEFN-------- } 278 \\ \text { GmFUS3 } & \text { PVET-FGSVEN-LSLDDFY-------- } 375 \\ \text { MtFUS3 } & \text { SVET-FGSVEN-LSLDDFY-------- } 314 \\ \text { VVFUS3 } & \text { ALEG-FGSVES-LSLDDFC-------- } 287 \\ \text { S1FUS3 } & \text { YPMPSFGSIEDSFSVDDFY-------- } 274\end{array}$

(a)

Fig. 2. (a) Alignment of FUS3 proteins from dicots. (b) Alignment of FUS3 proteins from monocots. Conserved sequences covered with green lines are B3 domains. Conserved sequences covered with dark blue lines are FUS3-specific fragments of dicots. Conserved sequences covered with light blue lines are FUS3-specific fragments of monocots. 


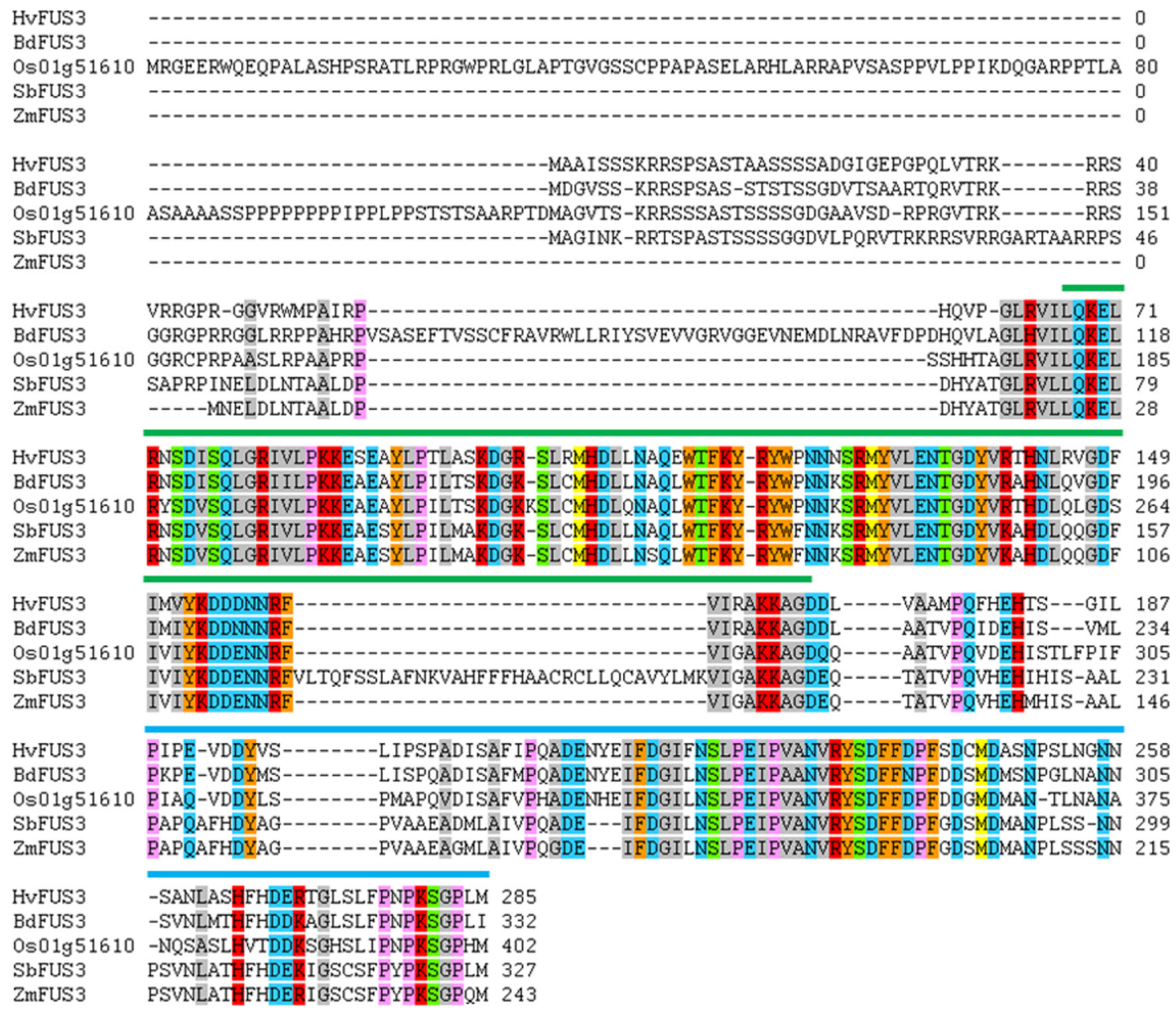

(b)

Fig. 2. (Continued)

\subsection{Analysis of conserved domains in AFL genes}

Comparison of the domain structure of proteins encoded by ABI3, FUS3 and LEC2 demonstrated that the homologous genes shared similar number and type of conserved domains. We also detected a decreasing trend in the number of domains contained in ABI3, FUS3 and LEC2. All ABI3 genes contain four domains, namely A1, B1, B2, and B3. Of them, the A1 domain is located at the N-terminus of the protein, which plays an important role in the function of these proteins as transcription activators. ${ }^{32}$ The B1 domain of ABI3 binds to the N-terminal charged domains of ABI5, and is critical for the physical interaction between ABI3 and ABI5. ${ }^{35}$ The $\mathrm{B} 2$ domain is required for trans-activation of the Em and $2 \mathrm{~S}$ promoter, ${ }^{36,37}$ thus being essential for regulation of other proteins. ${ }^{38}$ The B3 domain is a common conserved domain existing in all AFL subfamily members. Comparison of gene structure revealed that the $\mathrm{A} 1$ and $\mathrm{B} 1$ domains have been lost in both FUS3 and LEC2 sequences. However, we detected a novel FUS3-specific domain at the C-terminal of the sequences that was highly conserved but showed significant difference between 
monocot and dicot plants (Fig. 2). Blast searches against Pfam 24.0 using the FUS3specific fragment as a query sequence returned no hits. Furthermore, only a single B3 domain was found in the LEC2 and IDEF homologous genes. The B3 domains were mostly located in the middle part of the $L E C 2$ sequences but in the proximal region of the $\mathrm{C}$ terminus of IDEF homologues.

\section{Discussion}

Much attention has been paid in recent years to the evolution of the B3 superfamily. ${ }^{1,2}$ The role of the AFL B3 domain transcription factors in controlling seed development has also been studied extensively. ${ }^{10}$ However, the evolutionary relationships of the AFL members have not yet been clearly assessed due to only a few sequences from model plants used in previous studies. We explored the evolutionary history of the AFL subfamily based on sequences collected from all available databases, covering all major lineages of land plants. The data shows clearly that the AFL genes underwent a series of independent duplications and diversifications during land plant evolution. The duplicate $A B I 3$ genes of non-seed plants seem to have structurally and functionally diverged prior to the diversification of seed plants, ${ }^{39,40}$ leading to the reduction of the numbers of $A B I 3$ in the seed plant genomes, and giving rise to the appearance of the FUS3 gene. In accompaniment to the divergence of gymnosperm and angiosperm ${ }^{41,42}$ about 300 million years ago, ${ }^{43}$ the FUS3 gene experienced a new round of duplication with subsequent diversification through neo- and/or sub-functionalization. As a result, one copy retained the original function of FUS3 in both gymnosperm and angiosperm genomes and the other copy diverged into the ancestors of $L E C 2$ and IDEF. Present data suggest that the $L E C 2$ gene appeared initially and limitedly distributed in the dicot genomes, ${ }^{5,44,45}$ while the functionally diverged $I D E F$ gene limited to the monocot genomes. ${ }^{34}$ Therefore, the ABI3, FUS3 and LEC2 genes have appeared in the land plant genomes in a stepwise pattern.

There is a clear trend toward the reduction of the conserved domain contained in the ABI3, FUS3 and LEC2 genes. Moreover, functional diversification of ABI3, FUS3, LEC2 and IDEF seems to be correlated with the variation in domain structure. It has been reported that ABI3 plays an important role in regulation of abscisic acid-responsive genes. Mutants of AtABI3 showed low sensitivity to ABA, chlorophyll breakdown and desiccation intolerance. ${ }^{3,6,8}$ Mutations of FUS3 and LEC2 lead to similar pleiotropic effects on seed phenotype, including desiccation intolerant and viviparous. ${ }^{9,46,47}$ In contrast, IDEF functions as the early response factor toward iron deficiency in cereals because it can recognize the CATGC sequence within IDE1 (Iron-Deficiency Element). ${ }^{33,34,48,49}$ Compared to the wide range of expression patterns of ABI3, FUS3 and LEC2 exhibit restricted spatial and temporal expression. ${ }^{50} A B I 3$ was found to express in the whole embryo throughout the maturation phase in Arabidopsis, ${ }^{51}$ and it also has been detected in vegetative organs. ${ }^{9,52}$ FUS3 was found to be highly expressed in siliques ${ }^{4}$ and protoderm $^{53}$ 
before the maturation phase, ${ }^{54}$ while $L E C 2$ was mainly expressed in the embryo axis. ${ }^{9}$ The restricted spatial and temporal expression of FUS3 and LEC2 possibly resulted from the reduction of conserved domains in these genes, which increases specialization of gene function.

ABI3, FUS3 and LEC2 are currently considered the master regulatory genes for seed maturation. ${ }^{9,10}$ Based on the currently available data, the $L E C$ 2 genes are only distributed in the dicot genomes. It is thus doubtful whether all the ABI3, FUS3 and $L E C 2$ genes are indispensable components of the regulatory network controlling seed development for all seed plants. To answer this question, we need more complete genome sequences, especially the genomic information of gymnosperms. Meanwhile, the evolutionary relationships between $L E C 2$ and IDEF remain to be further assessed. It is currently unsure when $L E C 2$ and $I D E F$ diverged. These two genes probably descended from different ancestors that appeared at the early stage of angiosperm diversification, with the $L E C 2$ and IDEF homologues sequences lost in the monocot and dicot genomes, respectively. But we also cannot exclude the possibility that IDEF was derived from $L E C 2$ through duplication and subsequent diversification during the monocots-dicots split. To investigate when these genes originated and how they diverged during evolution will provide crucial insights into the molecular mechanisms controlling seed formation and evolution.

\section{Acknowledgments}

This work was supported by the National Natural Science Foundation of China (30870177).

\section{References}

1. Swaminathan K, Peterson K, Jack T, The plant B3 superfamily, Trends Plant Sci 13:647-655, 2008.

2. Romanel E, Schrago CG, Counago RM, Russo C, Alves-Ferreira M, Evolution of the B3 DNA binding superfamily: New insights into REM family gene diversification, Plos One 4:e57916, 2009.

3. Ooms J, Leonkloosterziel KM, Bartel D, Koornneef M, Karssen CM, Acquisition of desiccation tolerance and longevity in seeds of arabidopsis-thaliana - A comparativestudy using abscisic acid-insensitive ABI3 mutants, Plant Physiol 102:1185-1191, 1993.

4. Luerssen K, Kirik V, Herrmann P, Misera S, FUSCA3 encodes a protein with a conserved VP1/ABI3-like B3 domain which is of functional importance for the regulation of seed maturation in Arabidopsis thaliana, Plant J 15:755-764, 1998.

5. Stone SL, Kwong LW, Yee KM, Pelletier J, Lepiniec L, Fischer RL, Goldberg RB, Harada JJ, LEAFY COTYLEDON2 encodes a B3 domain transcription factor that induces embryo development, Proc Natl Acad Sci USA 98:11806-11811, 2001.

6. Meinke DW, Franzmann LH, Nickle TC, Yeung EC, Leafy cotyledon mutants of arabidopsis, Plant Cell 6:1049-1064, 1994.

7. Parcy F, Valon C, Kohara A, Misera S, Giraudat J, The ABSCISIC ACIDINSENSITIVE3, FUSCA3, and LEAFY COTYLEDON1 loci act in concert to control multiple aspects of Arabidopsis seed development, Plant Cell 9:1265-1277, 1997. 
8. Meurs C, Basra AS, Karssen CM, Vanloon LC, Role of abscisic-acid in the induction of desiccation tolerance in developing seeds of arabidopsis-thaliana, Plant Physiol 98:1484-1493, 1992.

9. To A, Valon C, Savino G, Guilleminot J, Devic M, Giraudat J, Parcy F, A network of local and redundant gene regulation governs Arabidopsis seed maturation, Plant Cell 18:1642-1651, 2006.

10. Santos-Mendoza M, Dubreucq B, Baud S, Parcy F, Caboche M, Lepiniec L, Deciphering gene regulatory networks that control seed development and maturation in Arabidopsis, Plant J 54:608-620, 2008.

11. Braybrook SA, Harada JJ, LECs go crazy in embryo development, Trends Plant Sci 13:624-630, 2008.

12. Steeves TA, The evolution and biological significance of seeds, Can $J$ Bot 61: 3550-3560, 1983.

13. West M, Harada JJ, Embryogenesis in higher-plants-An overview, Plant Cell 5: 1361-1369, 1993.

14. Goldberg RB, Depaiva G, Yadegari R, Plant embryogenesis - Zygote to seed, Science 266:605-614, 1994.

15. Harada JJ, Role of Arabidopsis LEAFY COTYLEDON genes in seed development, J Plant Physiol 158:405-409, 2001.

16. Vicente-Carbajosa J, Carbonero P, Seed maturation: Developing an intrusive phase to accomplish a quiescent state, Int J Dev Biol 49:645-651, 2005.

17. Mccarty DR, Hattori T, Carson CB, Vasil V, Lazar M, Vasil IK, The VIVIPAROUS1 developmental gene of maize encodes a novel transcriptional activator, Cell 66: 895-905, 1991.

18. Xie ZY, Li X, Glover BJ, Bai SN, Rao GY, Luo JC, Yang J, Duplication and functional diversification of HAP3 genes leading to the origin of the seed-developmental regulatory gene, LEAFY COTYLEDON1 (LEC1), in nonseed plant genomes, Mol Biol Evol 25:1581-1592, 2008.

19. Lespinet O, Wolf YI, Koonin EV, Aravind L, The role of lineage-specific gene family expansion in the evolution of eukaryotes, Genome Res 12:1048-1059, 2002.

20. Zhang YJ, Wang LJ, The WRKY transcription factor superfamily: Its origin in eukaryotes and expansion in plants, BMC Evol Biol 5(1):1, 2005.

21. Guo AY, Zhu QH, Gu XC, Ge S, Yang J, Luo JC, Genome-wide identification and evolutionary analysis of the plant specific SBP-box transcription factor family, Gene 418:1-8, 2008.

22. Huang XQ, Madan A, CAP3: A DNA sequence assembly program, Genome Res 9: 868-877, 1999.

23. Altschul SF, Madden TL, Schaffer AA, Zhang JH, Zhang Z, Miller W, Lipman DJ, Gapped BLAST and PSI-BLAST: A new generation of protein database search programs, Nucleic Acids Res 25:3389-3402, 1997.

24. Thompson JD, Gibson TJ, Plewniak F, Jeanmougin F, Higgins DG, The CLUSTAL_X windows interface: Flexible strategies for multiple sequence alignment aided by quality analysis tools, Nucleic Acids Res 25:4876-4882, 1997.

25. Larkin MA, Blackshields G, Brown NP, Chenna R, McGettigan PA, McWilliam H, Valentin F, Wallace IM, Wilm A, Lopez R, Thompson JD, Gibson TJ, Higgins DG, Clustal W and Clustal X version 2.0, Bioinformatics 23:2947-2948, 2007.

26. Hall TA, BioEdit: A user-friendly biological sequence alignment editor and analysis program for Windows 95/98/NT, Nucleic Acids Symp Ser 41:95-98, 1999.

27. Tamura K, Dudley J, Nei M, Kumar S, MEGA4: Molecular Evolutionary Genetics Analysis (MEGA) software version 4.0, Molecular Biology and Evolution 24: 1596-1599, 2007. 
28. Castresana J, Selection of conserved blocks from multiple alignments for their use in phylogenetic analysis, Mol Biol Evol 17:540-552, 2000.

29. Swofford DL, PAUP - A computer-program for phylogenetic inference using maximum parsimony, J Gen Physiol 102:A9, 1993.

30. Jones DT, Taylor WR, Thornton JM, The rapid generation of mutation data matrices from protein sequences, Computer Applications in the Biosciences 8:275-282, 1992.

31. Stamatakis A, RAXML-VI-HPC, Maximum likelihood-based phylogenetic analyses with thousands of taxa and mixed models, Bioinformatics 22:2688-2690, 2006.

32. Marella HH, Sakata Y, Quatrano RS, Characterization and functional analysis of ABSCISIC ACID INSENSITIVE3-like genes from Physcomitrella patens, Plant $J$ 46:1032-1044, 2006.

33. Kobayashi T, Ogo Y, Itai RN, Nakanishi H, Takahashi M, Mori S, Nishizawa NK, The transcription factor IDEF1 regulates the response to and tolerance of iron deficiency in plants, Proc Natl Acad Sci USA 104:19150-19155, 2007.

34. Kobayashi T, Itai RN, Ogo Y, Kakei Y, Nakanishi H, Takahashi M, Nishizawa NK, The rice transcription factor IDEF1 is essential for the early response to iron deficiency, and induces vegetative expression of late embryogenesis abundant genes, Plant $J$ 60:948-961, 2009.

35. Nakamura S, Lynch TJ, Finkelstein RR, Physical interactions between ABA response loci of Arabidopsis, Plant J 26:627-635, 2001.

36. Marella HH, Quatrano RS, The B2 domain of VIVIPAROUS1 is bi-functional and regulates nuclear localization and transactivation, Planta 225:863-872, 2007.

37. Bies-Etheve N, Conceicao AD, Giraudat J, Koornneef M, Leon-Kloosterziel K, Valon C, Delseny M, Importance of the B2 domain of the Arabidopsis ABI3 protein for Em and 2S albumin gene regulation, Plant Mol Biol 40:1045-1054, 1999.

38. Hill A, Nantel A, Rock CD, Quatrano RS, A conserved domain of the viviparous-1 gene product enhances the DNA binding activity of the bZIP protein EmBP-1 and other transcription factors, J Biol Chem 271:3366-3374, 1996.

39. Crane PR, Phylogenetic analysis of seed plants and the origin of angiosperms, Ann Mo Bot Gard 72:716-793, 1985.

40. Chase MW, Soltis DE, Olmstead RG, Morgan D, Les DH, Mishler BD, Duvall MR, Price RA, Hills HG, Qiu YL et al., Phylogenetics of seed plants - an analysis of nucleotide-sequences from the plastid gene RBCL, Ann Mo Bot Gard 80:528-580, 1993.

41. Doyle JA, Donoghue MJ, Seed plant phylogeny and the origin of angiosperms - An experimental cladistic approach, Bot Rev 52:321-431, 1986.

42. Crane PR, Friis EM, Pedersen KR, The origin and early diversification of angiosperms, Nature 374:27-33, 1995.

43. Bowe LM, Coat G, DePamphilis CW, Phylogeny of seed plants based on all three genomic compartments: Extant gymnosperms are monophyletic and Gnetales' closest relatives are conifers, Proc Natl Acad Sci USA 97:4092-4097, 2000.

44. Tuskan GA, DiFazio S, Jansson S, Bohlmann J, Grigoriev I, Hellsten U, Putnam N, Ralph S, Rombauts S, Salamov A et al., The genome of black cottonwood, Populus trichocarpa (Torr. \& Gray), Science 313:1596-1604, 2006.

45. Malik MR, Wang F, Dirpaul JM, Zhou N, Polowick PL, Ferrie A, Krochko JE, Transcript profiling and identification of molecular markers for early microspore embryogenesis in Brassica napus, Plant Physiol 144:134-154, 2007.

46. Baumlein H, Misera S, Luerssen H, Kolle K, Horstmann C, Wobus U, Muller AJ, The FUS3 gene of arabidopsis-thaliana is a regulator of gene-expression during late embryogenesis, Plant J 6:379-387, 1994. 
47. Keith K, Kraml M, Dengler NG, Mccourt P, FUSCA3 - A heterochronic mutation affecting late embryo development in arabidopsis, Plant Cell 6:589-600, 1994.

48. Reidt W, Wohlfarth T, Ellerstrom M, Czihal A, Tewes A, Ezcurra I, Rask L, Baumlein $\mathrm{H}$, Gene regulation during late embryogenesis: The RY motif of maturation-specific gene promoters is a direct target of the FUS3 gene product, Plant $J$ 21:401-408, 2000.

49. Monke G, Altschmied L, Tewes A, Reidt W, Mock HP, Baumlein H, Conrad U, Seed-specific transcription factors ABI3 and FUS3: Molecular interaction with DNA, Planta 219:158-166, 2004.

50. Braybrook SA, Stone SL, Park S, Bui AQ, Le BH, Fischer RL, Goldberg RB, Harada JJ, Genes directly regulated by LEAFY COTYLEDON2 provide insight into the control of embryo maturation and somatic embryogenesis, Proc Natl Acad Sci USA 103:3468-3473, 2006.

51. Parcy F, Valon C, Raynal M, Gaubiercomella P, Delseny M, Giraudat J, Regulation of gene-expression programs during Arabidopsis seed development - Roles of the ABI3 locus and of endogenous ABSCISIC-ACID, Plant Cell 6:1567-1582, 1994.

52. Suzuki M, McCarty DR, Functional symmetry of the B3 network controlling seed development, Curr Opin Plant Biol 11:548-553, 2008.

53. Rohde A, Kurup S, Holdsworth M, ABI3 emerges from the seed, Trends Plant Sci 5:418-419, 2000.

54. Tsuchiya Y, Nambara E, Naito S, McCourt P, The FUS3 transcription factor functions through the epidermal regulator TTG1 during embryogenesis in Arabidopsis, Plant $J$ 37:73-81, 2004.

Yang Li received her Bachelor's degree in Ecology from Nanjing Agricultural University, China. She is currently pursuring her Master's degree in Botany in the School of Life Sciences, Fudan University.

Ke Jin received his M.Sc. degree in Software Engineering from Jilin University, China, and his Ph.D. degree in Bioinformatics from Fudan University, China, in 2010. He is currently a Postdoctoral Fellow in the Donnelly Centre for Cellular and Biomolecular Research in the Faculty of Medicine.

Zhu Zhu received her B.Sc. degree in Biology and Ph.D. degree in Plant Sciences from Xiamen University, China, in 2003 and 2009, respectively. She was a joint Ph.D. student at the University of California, Davis, from September 2007 to May 2009. She is currently working as a research assistant in the School of Life Sciences, Fudan University.

Ji Yang is a Professor of Evolutionary Biology at Fudan University. He received his B.Sc. (1983) in Biology from Beijing Normal University and M.Sc. (1986) in Botany from Peking University. He is interested in exploring the molecular basis of the major innovations that occurred during land plant evolution by studying the duplication, diversification and co-option of key developmental regulator genes. 